\title{
COMPARISON OF CHAIR SIDE TIME AND TREATMENT OUTCOMES OF STABILIZATION SPLINTS OBTAINED FROM DIGITAL VERSUS CONVENTIONAL WORKFLOW
}

\author{
Amr H. Elkhadem* and Reem H. Hossameldin**
}

\begin{abstract}
Introduction: The use of stabilization repositioning splints (SRS) to treat muscular TMDs is a growing practice owing to its' effectiveness and simplicity. The conventional construction procedure requires meticulous occlusal adjustments of the intra orally modified cold cured resin which consumes considerable chairside time. Changing the procedure to a full digital approach by using intraoral scan and CAD CAM workflow might decrease the chair side time. Aim: To evaluate the chairside time required to construct SRS with conventional impression procedure compared to intra oral scanning and printing. The improvement in pain scale and range of jaw opening in both groups will be also evaluated.
\end{abstract}

Materials and methods: 30 patients suffering from muscular TMDs were allocated into 2 groups. Group 1 received SRS made out of a vacuum sheet formed over a dental model and adjusted intra orally with cold cured resin. Group 2 received SRS made from intra oral scans and digitally constructed and 3D printed. The time needed in the impression appointment and SRS fitting appointment was calculated. Patients were followed for 8 weeks to evaluate improvement of pain scores and range of mandibular opening.

Results: Regarding the treatment time the impression appointment time was statistically insignificant for both groups $(13.15 \pm 1.77)$ and $(12.47 \pm 1.96)$ minutes for groups 1 and 2 respectively. For the fitting appointment the duration showed statistical significant decrease when comparing group $2(4.49 \pm 1.42 \mathrm{~min})$ to group $1(36.51 \pm 6.21 \mathrm{~min})$. For both group there was a statistical improvement in pain score and maximum incisal opening, yet there was no statistical significant difference between group 1 and 2 .

Conclusion: Construction SRS with intra oral scans yielded the same patient improvement when compared to conventional construction technique but with significant decrease in the required chairside time

* Associate Professor, Prosthodontics, Faculty of Dentistry, Cairo University

** Associate Professor, Oral and Maxillofacial Surgery, Faculty of Dentistry, Cairo University 


\section{INTRODUCTION}

Temporomandibular disorders (TMD) are clinical hinders affecting the temporomandibular joint (TMJ), myofascial muscles and related structures $^{1}$. There is currently no unified standard for the classification of TMD, but research diagnostic criteria for temporomandibular disorders (RDC/ TMD) are the most commonly applied criteria $^{2}$. The main clinical presentation involve TMJ pain and noise, myofascial pain, abnormal jaw function and abnormal or limited jaw movement ${ }^{3}$. TMD constitute a major problem, as they are a main source of chronic orofacial pain, interfering with daily activities and jaw functions ${ }^{4}$. These disorders are also commonly associated with other head and neck presentations, including headache, tinnitus, ear pain, neck and cervical spine dysfunction $^{5,6}$. Signs of TMD are detected in about $60-70 \%$ of the general population, yet only about one in four people with signs are actually presented with symptoms ${ }^{7}$. The etiologies of TMD are not yet clear, but contributing factors include macrotrauma, microtrauma, parafunctional habits and psychological elements ${ }^{8}$, systemic autoimmune diseases, occlusal abnormalities ${ }^{9}$, indirect trauma and hormonal and microbial factors. ${ }^{10}$ The main treatment options for most TMD include occlusal therapy including rehabilitation in indicated cases and oral appliances ${ }^{11,12}$, psychotherapy ${ }^{13}$, physical therapy ${ }^{14}$, medications ${ }^{15}$, manual therapy ${ }^{16,17}$, and surgeries ${ }^{18}$. In practice, the occlusal splint is a removable appliance, usually fabricated of resin and most often designed to cover all of the occlusal and incisal surfaces of the teeth in the upper or lower jaw. Occlusal splint therapy is most commonly used clinical approach because of its ease of use, low cost, and broad indications. A previous meta-analysis addressed the effectiveness of splint therapy for TMD, but why it is effective remains unclear ${ }^{19}$. The effectiveness of splint therapy in TMD patients in a meta-analysis of published results was examined and results indicated that splint therapy effectively reduces pain levels in TMD patients, and reduces the frequency of pain in patients with TMJ clicking. Additionally, splint therapy increased mouth opening ability in patients with initial MMO $<45 \mathrm{~mm} .{ }^{20-22}$

The conventional workflow for making splints includes maxillary and mandibular impression. Over the produced models hard vacuum formed clear template is made. The template occlusal surface is modified intra-orally with cold cured transparent resin by making an anterior deprogramming jig with centric condylar position and minimal posterior separation. A second resin mix is added posterior to create even occlusal centric contacts with bilateral canine guidance. The procedure is time consuming, and requires patient cooperation and tolerance to resin irritation. Furthermore, the deprogramming over resin intra orally is relatively difficult and require high operator experience ${ }^{21}$. The introduction of modern intra oral scanners was introduced to provide a fast an accurate way to record 3D arch form and jaw relationship in a reproducible way. Full arch scans became a reliable and accurate protocols is different appliance therapies as orthodontic aligners $^{23}$ and digital wax up ${ }^{24}$. The data on using a complete digital workflow to fabricate TMJ bite splints is still not well clarified in the literature.

\section{MATERIALS AND METHODS}

\section{Sampling and grouping:}

Thirty patients were selected from the outpatient clinic in the faculty of dentistry, Cairo university, diagnosed with TMD pain of muscular origin. The patients were randomly categorized into 2 groups using sealed envelopes. Group1 received stabilization repositioning splint (SRS) with conventional impression and vacuum formed splints modified intra orally with cold cured resin. Group 2 received SRS made by intra oral scan and digitally designed to desired occlusal contacts and 3D printed. The patients approved to participate in 
the study. The operator and assessor could not be blinded due to the nature of the treatment.

\section{Patient examination and evaluation}

Preoperative and postoperative clinical assessments were performed on all patients, including patient history and a physical examination. The chief complaint, history of present illness, history of medications, imaging assessment (panoramic radiography, MRI), maximum inter incisal opening (MIO), direct and indirect joint loading, joint noise (click, crepitation), and an assessment of muscle and joint pain were recorded. A visual analog scale (VAS) ranging from 0 to100 (0 representing no pain/proper function and 100 representing maximum pain/dysfunction) was used to score pain and function at the first visit and last visit. These clinical assessments were analyzed to determine the outcome in regards to success or failure. The primary outcome variable of success or failure was determined by the reduction in joint pain postoperatively. Secondary outcome variables included joint function, MIO, need for medications, joint loading sign, and muscle pain.

\section{SRS construction:}

\section{Group 1:}

During the impression appointment and maxillary and mandibular impression were made using regular set alginate impression material (Tropicalgin, Zermach, Germany). Care was taken to obtain and impression with at least $5 \mathrm{~mm}$ extension below the gingival margins and distal extension beyond the last molar tooth. The impressions were poured into type III dental stone.

Over the maxillary models, a $1 \mathrm{~mm}$ thick vacuum formed sheet was thermally adapted under vacuum pressing machine (Bioart, brazil). The vacuum sheet was trimmed with a scissor at the gingival margin level. The vacuum sheet was tried for proper seating and retention.
Transparent cold cured resin (Acrostone, Egypt) was mixed to a dough consistency and placed over the palatal area of the vacuum template at the upper incisors. The patient was guided to close in centric relation using bimanual manipulation technique to seat the condylar disc complex in the antero-superior position. The patient was asked to closed just before the first posterior contact between the vacuum template the and lower posterior teeth. When the cold cured resin set, the excess was trimmed with an acrylic burr and the centric position verified inside the patient mouth assuring repeatable closure on the set anterior jig. A second mix of cold cured resin was made to dough consistency and applied posteriorly bilaterally over the vacuum template. The patient was asked to close till the lower teeth contact the set anterior jig.

After complete setting of the resin the excess is trimmed and all indentations of the buccal and lingual slopes of the lower teeth were trimmed keeping only the cuspal tip contact. The acrylic slope at the canine area was kept. Inside the patient mouth an articulating paper was used to eliminate inferences and prematurity. The patient was asked to slide laterally and excessive contact were removed so that the lower canines glides smoothly over he acrylic in the upper canine region (canine guidance)

\section{Group 2:}

During the impression appointment the upper and lower arches were scanned with an intra oral scanner (Medit i500, South Korea). An anterior jig was constructed using green stick compound. The green stick was softened and adapted to cover the upper 4 incisors. The top surface was made flat. The patient was guided to close in centric position using bimanual manipulation. The vertical stopper was adjusted to guarantee at least $1 \mathrm{~mm}$ posterior separation at the last molar. The jig was verified to assure repeated centric closure. With the patient biting on the compound jig the jaw relation is scanned bilaterally (Figure 1). 
The scans were manipulated using Blender for Dental software (B4D, Australia). Initially the upper model was surveyed to select the most suitable path of insertion. All undercuts above 0.25 $\mathrm{mm}$ were blocked digitally to obtain a selective block out model for splint construction (Figure 2). The splint was designed to cover the maxillary teeth up to the gingival margin. The occlusal top surface of the splint was extruded to make imprints in the lower model cusp tips and incisal edges.

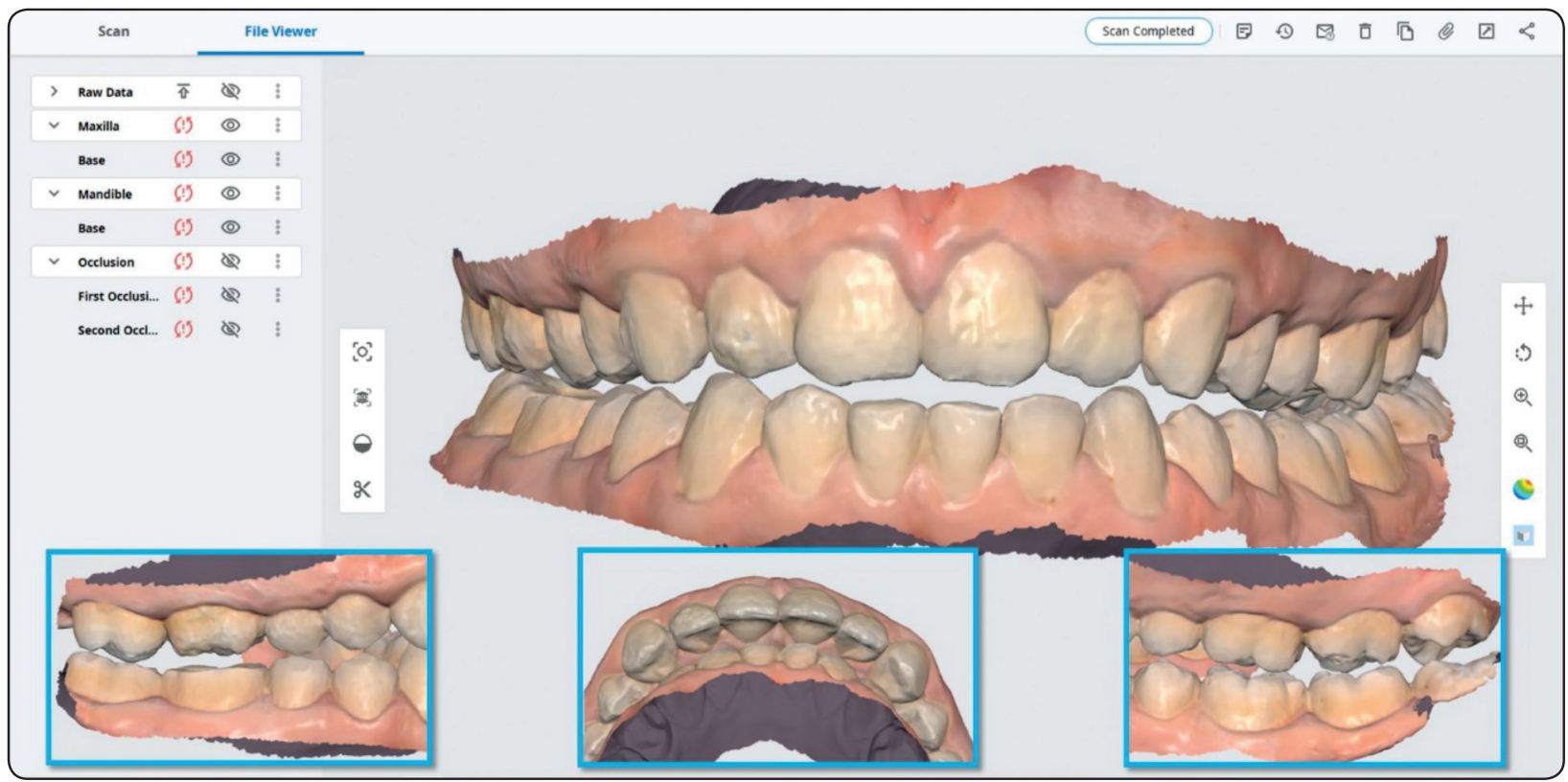

Fig. (1) Interface of Medit I 500 acquisition software showing maxillary and mandibular scan with inter-occlusal record made at minimal posterior disclusion

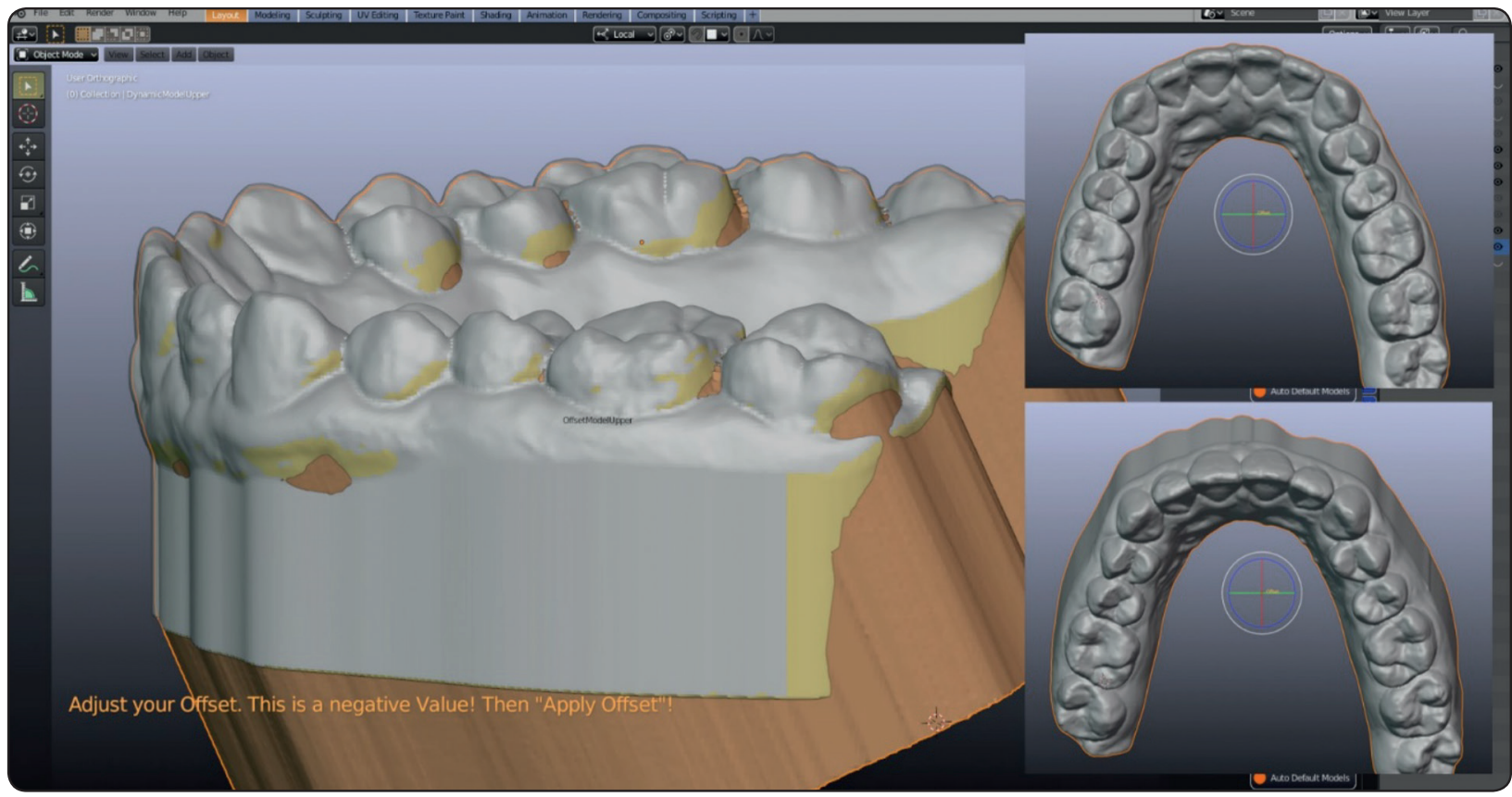

Fig. (2) Virtual blockout on the Blender for dental software. Digital surveying for a suitable path of insertion and block out (brown) leaving $0.25 \mathrm{~mm}$ undercuts (yellow) 
The lower model was used to cut the teeth imprints with 200 microns tolerance gap (Figure 3). The finished splint was printed with tooth colored resin at 100 micron printing layer thickness with horizontal orientation on the printing platform (Phrozen shuffle XL, Taiwan). The printed guide was processed by cleaning in isopropyl alcohol and light curing for 20 minutes in light curing box (Pressing Dental, Italy). Finally, the buccal and lingual slope of the lower teeth imprints were grinded and the splint finished and polished. During the fitting appointment the seating and retention of the splint were checked. Occlusal contacts were checked with articulating paper to assure even centric contact and unobstructed lateral sliding with canine guidance.

For both groups time lapsed during the impression visit and fitting visit was calculated with a stop watch. At the fitting visit the pain score was recorded using visual analogue scale (VAS with scale from 0-100). Additionally, the maximal incisal opening (MIO)was measured using a digital caliper. After 8 weeks the patient was recalled and the VAS and MIO were recorded.

\section{RESULTS}

The study evaluated the chairside time required for the fabrication of the SRS in both group (Figure $4)$. The mean time for the records visit for Groups 1 and 2 were $13.15 \pm 1.77$ and $12.47 \pm 1.96$ respectively. The student $t$ test showed there was no statistical significant difference between both groups $(\mathrm{P}=0.323446)$. For the splint fitting visit the mean chairside time was $36.51 \pm 6.21$ minutes for group 1 compared to $4.49 \pm 1.42$ minutes for group 2 . The statistical analysis showed a statistical significant reduction in the chairside for group $2(\mathrm{P}<0.001)$ (Mean difference $=32.02 \pm 1.645,95 \%$ Confidence Interval 28.65, 35.39)

Regarding the Pain score both groups showed significant decrease in the pain scores when comparing the fitting appointments and after 8 weeks $\mathrm{P}<0.01$ (Figure 5). For group 1, the mean value for the pain score dropped from $72.73 \pm 10.45$ to $31.67 \pm 8.38$. Similarly, in group 2 , the mean value for the pain score dropped from $69.13 \pm 10.12$ to $27.67 \pm 8.42$. When comparing the changes in the pain score in both group after 8 weeks there was no statistical significant difference between both groups $\mathrm{P}=0.203$

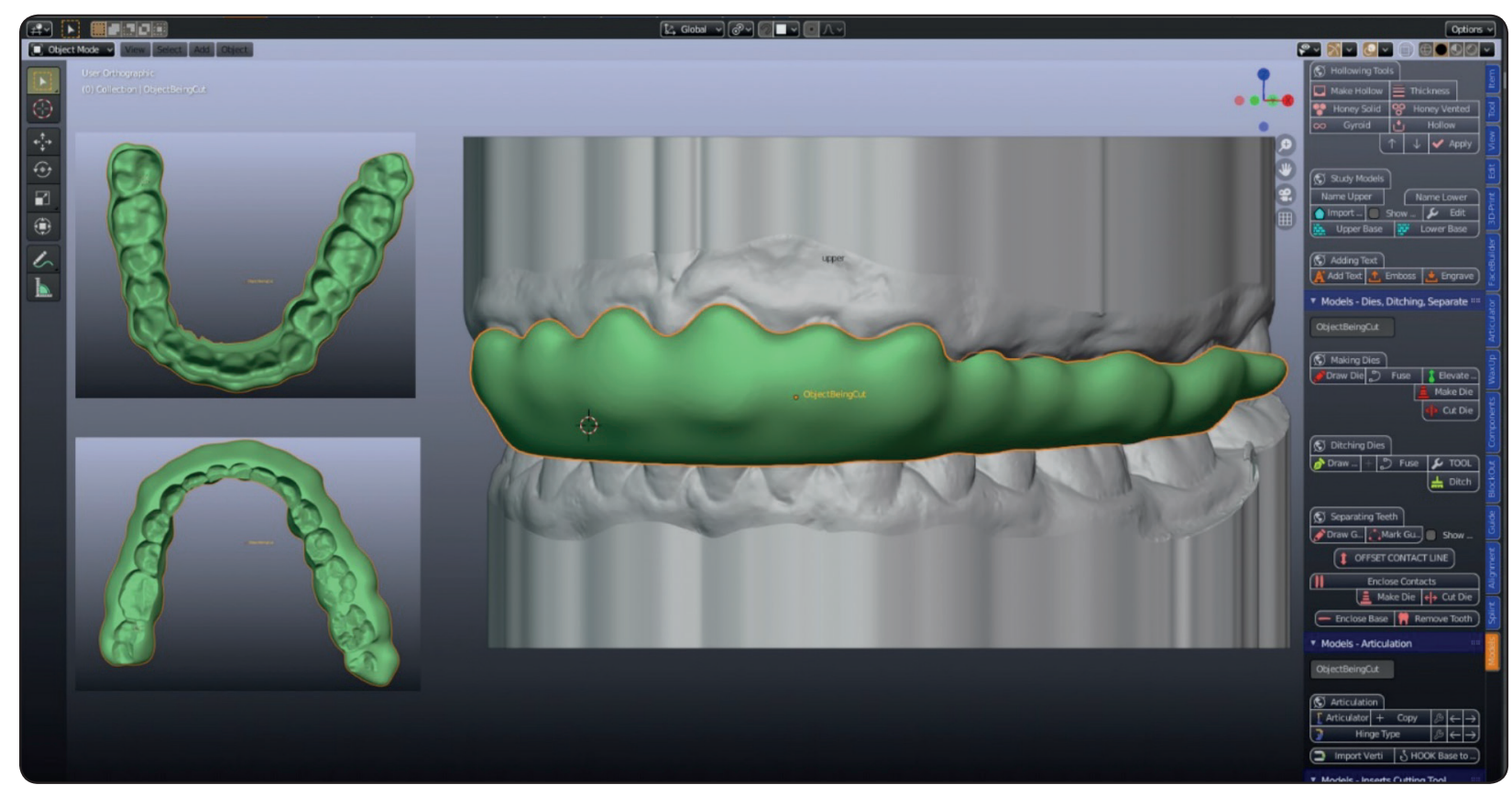

Fig. (3): SRS design. The shape of the fitting surface and imprints of the opposing were made using Boolean cutting tool 
Regarding the changes of the maximal incisal opening in both groups, both group showed increase in the MIO values by time (Figure 6 ). For group 1 , and group 2 the paired $\mathrm{T}$ test showed significant increase in MOI $(\mathrm{P}<0.01)$ when comparing fitting day and after 8 weeks. For group 1, the mean value for MIO increased from $30.93 \pm 4.20$ to $36.47 \pm 3.81 \mathrm{~mm}$. For group 2, the mean value for MIO also increased from $29.93 \pm 2.99$ to $36.93 \pm 2.71 \mathrm{~mm}$. When comparing the changes in MIO in both groups after using the splint for 8 weeks there was statistically insignificant difference between the two groups $\mathrm{P}=0.702$.



Fig. (4): Chairside time values (minutes) for conventional versus digital workflow

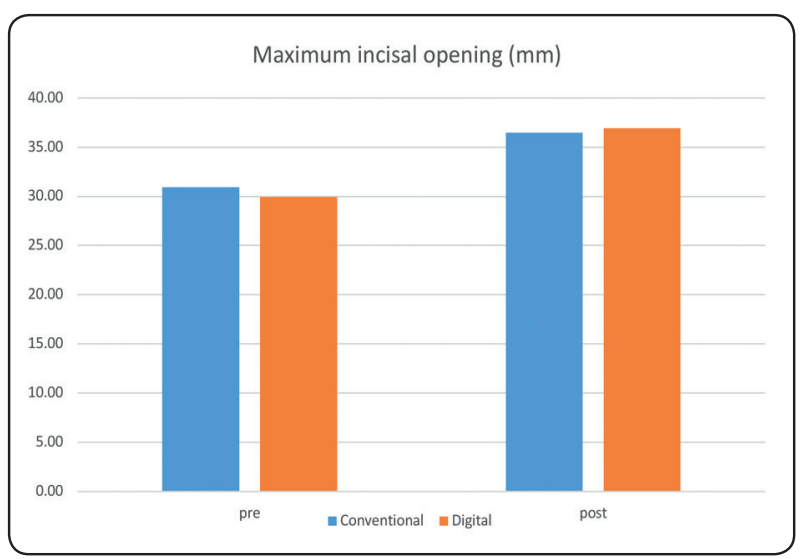

Fig. (5): Mean values of Maximum incisal opening (mm) in conventional versus digital workflow

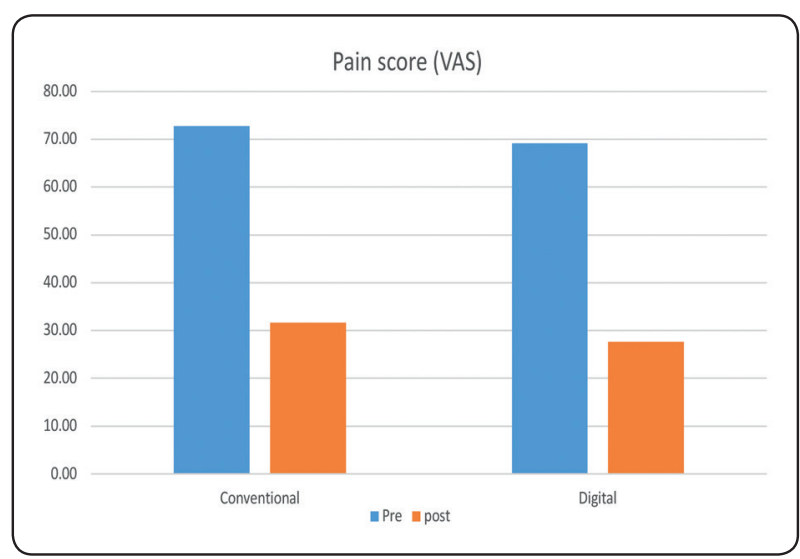

Fig. (6): Mean values of pain scores in conventional versus digital workflow

\section{DISCUSSION}

The use of intraoral scanners is becoming a popular and growing application in the dental field. The scanners are believed to improve the workflow in terms of consistent results and reduction of chairside time. The present study aimed to investigate the chairside time elapsed to fabricate SRS with both conventional and digital approaches. It is worthy to note that the time lapse for making impressions and in making scans is a function of multiple factors as patient cooperation, operator experience and learning curve. Additionally, impression material working time and scanner speed might also influence the overall time. In a study performed by Glisic et $\mathrm{al}^{25}$, they evaluated the chairside time lapsed when making conventional alginate impression versus IO scans in young adolescent patients. They found that the time was similar in both groups with an average of 11-12 minute with no statistical difference between the two groups. In the present study the chairside time in both groups was around 13 minutes with no statistical difference. The intra oral scan time was slightly longer. This can be explained by the fact that in the IO scan group the time lapse is made for scanning both arches and make virtual bite scan after making a scan jig. While in the conventional group the time was completely lapsed for maxillary and mandibular impressions with no jaw relation 
records. When comparing the fitting appointment time, the digital workflow significantly reduced the time by almost $85 \%$. The main cause behind such decrease is that the occlusal equilibration in the IO scan group was done on the software based on a digital jaw record registration. Also, the virtual block out with controlled selection of undercut guarantee the fit and retention of all produced splints. The time lapse in the conventional group can be attributed to the need to register the jaw relation on cold cured resin on 2 steps and the time lapse to grind off and finish the excess. Such maneuver is totally eliminated with the digital workflow. When an intraoral scanner is not available a hybrid work flow of using conventional impression with lab model scanning can help in reaching the same result by design the SRS over the laboratory scanned model $^{26}$.

It is worthy to note that the clinical outcomes of both techniques in regards of pain improvement and increase in the maximal incisal opening was comparable. This can be explained by the fact that both final splints exhibit the same fit quality and similar occlusal contact principals.

\section{CONCLUSION}

The digital workflow for fabricating stabilization splints significantly reduced the chairside time specially the time lapsed in the splint fitting session. Although Intra oral scanning did not reduce the chairside time in the impression visit compared to the physical impression, it indirectly affected the overall procedure time by providing a timely articulated digital models ready for splint design.

\section{REFERENCES}

1. Chen YW, Chiu YW, Chen CY, Chuang SK. Botulinum toxin therapy for temporomandibular joint disorders: A systematic review of randomized controlled trials. Int J Oral Maxillofac Surg. Published online 2015. doi:10.1016/j.ijom.2015.04.003
2. Dworkin SF, LeResche L. Research diagnostic criteria for temporomandibular disorders: review, criteria, examinations and specifications, critique. J Craniomandib Disord. Published online 1992.

3. Dworkin SF, Huggins KH, LeResche L, et al. Epidemiology of signs and symptoms in temporomandibular disorders: clinical signs in cases and controls. J Am Dent Assoc. Published online 1990. doi:10.14219/jada.archive.1990.0043

4. Armijo-Olivo S, Pitance L, Singh V, Neto F, Thie N, Michelotti A. Effectiveness of manual therapy and therapeutic exercise for temporomandibular disorders: Systematic review and meta-analysis. Phys Ther. Published online 2016. doi:10.2522/ptj.20140548

5. Gremillion HA. The prevalence and etiology of temporomandibular disorders and orofacial pain. Tex Dent J. Published online 2000 .

6. De Wijer A, De Leeuw JRJ, Steenks MH, Bosman F. Temporomandibular and cervical spine disorders: Self-reported signs and symptoms. Spine (Phila Pa 1976). Published online 1996. doi:10.1097/00007632199607150-00005

7. Graber, Rakosi P. Dentofacial Orthopedics with Functional Appliances. In: 2nd ed. St. Louis: Mosby; 2009:135-140.

8. Korioth TWP, Hannam AG. Effect of bilateral asymmetric tooth clenching on load distribution at the mandibular condyles. J Prosthet Dent. Published online 1990. doi:10.1016/0022-3913(90)90154-5

9. Nitzan DW. Intraarticular pressure in the functioning human temporomandibular joint and its alteration by uniform elevation of the occlusal plane. J Oral Maxillofac Surg. Published online 1994. doi:10.1016/02782391(94)90476-6

10. Sharma S, Pal U, Gupta D, Jurel S. Etiological factors of temporomandibular joint disorders. Natl J Maxillofac Surg. Published online 2011 doi:10.4103/0975-5950.94463

11. Wright E, Anderson G, Schulte J. A randomized clinical trial of intraoral soft splints and palliative treatment for masticatory muscle pain. J Orofac Pain. Published online 1995.

12. Türp JC, Komine F, Hugger A. Efficacy of stabilization splints for the management of patients with masticatory muscle pain: a qualitative systematic review. Clin Oral Investig. Published online 2004. doi:10.1007/s00784-0040265-4 
13. Systematic review and meta-analysis of randomized controlled trials evaluating intraoral orthopedic appliances for temporomandibular disorders. In: Evidence-Based Orthodontics. ; 2018. doi:10.1002/9781119289999.oth51

14. Ahrari F, Madani AS, Ghafouri ZS, Tunér J. The efficacy of low-level laser therapy for the treatment of myogenous temporomandibular joint disorder. Lasers Med Sci. Published online 2014. doi:10.1007/s10103-012-1253-6

15. Pramod G V., Shambulingappa P, Shashikanth MC, Lele $\mathrm{S}$. Analgesic efficacy of diazepam and placebo in patients with temporomandibular disorders: A double blind randomized clinical trial. Indian J Dent Res. Published online 2011. doi:10.4103/0970-9290.87062

16. Brehnan K, Boyd RL, Laskin J, Gibbs CH, Mahan P. Direct Measurement of Loads at the Temporomandibular Joint in Macaca arctoides. J Dent Res. Published online 1981. doi:10.1177/00220345810600101501

17. Tuncer AB, Ergun N, Tuncer AH, Karahan S. Effectiveness of manual therapy and home physical therapy in patients with temporomandibular disorders: A randomized controlled trial. J Bodyw Mov Ther. Published online 2013. doi:10.1016/j.jbmt.2012.10.006

18. Al-Moraissi EA. Arthroscopy versus arthrocentesis in the management of internal derangement of the temporomandibular joint: A systematic review and metaanalysis. Int J Oral Maxillofac Surg. Published online 2015. doi:10.1016/j.ijom.2014.07.008

19. Al Quran FAM, Kamal MS. Anterior midline point stop device (AMPS) in the treatment of myogenous TMDs: Comparison with the stabilization splint and control group. Oral Surgery, Oral Med Oral Pathol Oral Radiol
Endodontology. 2006;101(6):741-747. doi:10.1016/j. tripleo.2005.04.021

20. Zhang C, Wu JY, Deng DL, et al. Efficacy of splint therapy for the management of temporomandibular disorders: A meta-analysis. Oncotarget. Published online 2016. doi:10.18632/oncotarget.13059

21. Greene CS, Menchel HF. The Use of Oral Appliances in the Management of Temporomandibular Disorders. Oral Maxillofac Surg Clin North Am. Published online 2018. doi:10.1016/j.coms.2018.04.003

22. Casares G, Thomas A, Carmona J, Acero J, Vila CN. Influence of oral stabilization appliances in intra-articular pressure of the temporomandibular joint. Cranio - J Craniomandib Pract. Published online 2014. doi:10.1179 /0886963413Z.00000000030

23. Kravitz ND, Groth C, Jones PE, Graham JW, Redmond WR. Intraoral digital scanners. J Clin Orthod. 2014;48(6): 337-47.

24. Zimmermann M, Mehl A. Virtual smile design systems: a current review. Int J Comput Dent. 2015;18(4):303-17.

25. Olja Glisic, Louise Hoejbjerre, and Liselotte Sonnesen. A comparison of patient experience, chair-side time, accuracy of dental arch measurements and costs of acquisition of dental models. Angle Orthod. 2019 Nov; 89(6): 868-875.

26. RS Algabri, AY Alqutaibi, AH Elkadem, EA Maher, AF Kaddah. Patient's satisfaction and muscles activity after management of temporomandibular disorders patients using computer-aided design/computer-aided manufacturing versus conventional occlusal splints (randomized clinical trial). International Dental \& Medical Journal of Advanced Research 3 (1), 1-8 